\author{
Jukka Takala \\ R. Philip Dellinger \\ Kati Koskinen \\ Arthur St. Andre \\ Martyn Read \\ Mitchell Levy \\ Stephan M. Jakob \\ Patricia Veiga C. Mello \\ Raymond Friolet \\ Esko Ruokonen
}

\section{Development and simultaneous application of multiple care protocols in critical care: a multicenter feasibility study}

Received: 21 September 2007

Accepted: 3 March 2008

Published online: 3 April 2008

(C) Springer-Verlag 2008

Electronic supplementary material

The online version of this article

(doi:10.1007/s00134-008-1084-x) contains supplementary material, which is available to authorized users.

Conflicts of interest: This trial was investigator initiated and received funding from TEKES (Finnish funding agency for technology and innovation), and from Deio (Helsinki, Finland) to cover part of the research personnel costs and the costs of data collection and statistical analysis. The software prototype was written by Deio based on specifications from the investigators. Author K.K. is an employee of Intensium (Kuopio, Finland), a company that managed the data collection and statistical analysis. No other conflicts of interest are declared.

These authors performed this study on behalf of the CPIC Study Group.

J. Takala ( $)$ S. M. Jakob · R. Friolet Bern University Hospital and University of Bern, Department of Intensive Care Medicine,

Freiburgstrasse, 3010 Bern, Switzerland e-mail: jukka.takala@insel.ch

Tel.: +41-31-6324144

Fax: +41-31-6324100
R. P. Dellinger

Cooper University Hospital, Critical Care

Division, Dorrance 393,

One Cooper Plaza, Camden, NJ, 08103, USA

K. Koskinen

Intensium Ltd.

Microkatu 1, 70211 Kuopio, Finland

A. St. Andre

Washington Hospital Center, Surgical

Critical Care, Rm 4B 42,

110 Irving Street NW,

Washington, DC, 20010, USA

M. Read

University Hospital of Wales, Critical Care

Services, Cardiff and Vale NHS Trust,

Heath Park, CF14 4XW Cardiff, UK

M. Levy

Rhode Island Hospital, Medical Intensive

Care Unit,

593 Eddy Street, Providence,

RI, 02906, USA

\section{P. V. C. Mello}

Universidade Estadual Piaui, Intensive Care Unit, Hospital de Terapia Intensiva,

2131 Anfrisio Lobao Teresina, 64041-280

Piaui, Brazil

E. Ruokonen

Kuopio University Hospital, Department of Intensive Care,

P.O. Box 1777, 70211 Kuopio, Finland
Abstract Objective: To test the feasibility of and interactions among three software-driven critical care protocols. Design: Prospective cohort study. Setting: Intensive care units in six European and American university hospitals. Patients: 174 cardiac surgery and 41 septic patients. Interventions: Application of software-driven protocols for cardiovascular management, sedation, and weaning during the first 7 days of intensive care. Measurements and results: All protocols were used simultaneously in $85 \%$ of the cardiac surgery and $44 \%$ of the septic patients, and any one of the protocols was used for 73 and $44 \%$ of study duration, respectively. Protocol use was discontinued in $12 \%$ of patients by the treating clinician and in $6 \%$ for technical/administrative reasons. The number of protocol steps per unit of time was similar in the two diagnostic groups (n.s. for all protocols). Initial hemodynamic stability (a protocol target) was achieved in $26 \pm 18 \mathrm{~min}$ (mean $\pm \mathrm{SD}$ ) in cardiac surgery and in $24 \pm 18 \mathrm{~min}$ in septic patients. Sedation targets were reached in $2.4 \pm 0.2 \mathrm{~h}$ in cardiac surgery and in $3.6 \pm 0.2 \mathrm{~h}$ in septic patients. 
Weaning protocol was started in 164 (94\%; 154 extubated) cardiac surgery and in 25 (60\%; 9 extubated) septic patients. The median (interquartile range) time from starting weaning to extubation (a protocol target) was 89 min (range 44-154 min) for the cardiac surgery patients and 96 min (range 56-205 min) for the septic patients. Conclusions: Multiple software-driven treatment protocols can be simultaneously applied with high acceptance and rapid achievement of primary treatment goals. Time to reach these primary goals may provide a performance indicator.

Keywords Cardiac surgery $\cdot$ Critical care - Disease management $\cdot$ Sedation $\cdot$ Treatment protocols $\cdot$ Weaning

\section{Introduction}

The increasing complexity of critical care processes and the variety of available diagnostic, monitoring, and interventional technologies present a challenge for providing the best possible care. Outside of health care, protocols have been used routinely for decades in the control of various complex processes in which safety, efficient resource utilization, and constant quality of the product have high priority. Typical examples include the aviation, nuclear power, petrochemical, and paper industries [1]. Despite the well-recognized complexity of critical care, systematic use of care protocols to control the delivery of critical care remains uncommon. When used, these protocols are rarely electronic. This is in sharp contrast to the wide application of software-driven process control outside of health care [1].

The potential for improving the quality of care by using protocols has been demonstrated in several recent studies in which single aspects of care delivery have been standardized. Such successes include sedation $[2,3]$, weaning from mechanical ventilation $[4,5]$, lung-protective mechanical ventilation $[6,7]$, goal-directed therapy in early sepsis [8] and in high-risk surgery [9, 10], and hemodynamic management after cardiac surgery $[11,12]$.

The areas of care where protocols have been proven useful are often closely interrelated; hence, softwaredriven process control using multiple protocols simultaneously would conceptually make sense, and using software to drive protocols at the bedside could facilitate their clinical application. However, even the application of single protocols in routine clinical practice outside clinical trials is associated with problems in compliance, acceptance, multiplication and generalizability $[13,14]$. Before there can be any evaluation of potential benefits from the simultaneous use of multiple protocols, the feasibility of this concept in the clinical routine should be assessed.

The aim of this international, multicenter study was to test in a clinical trial the feasibility and interactions among three software-driven critical care protocols (cardiovascular management, sedation, and weaning). The potential areas of protocolized care were defined, the rates of events triggering their use were assessed, and the protocols were developed by the study participants.

\section{Materials and methods}

The study protocol was approved by the ethics committee or institutional review board of each of the participating centers. The study was conducted from late 2002 to mid-2003. Written informed consent was obtained from the cardiac surgical patients (in one center the need for informed consent was waived). In septic patients, either the need for informed consent was waived or deferred consent was used.

Prior to the clinical study, potential protocol areas were identified by centers and in expert meetings using a modified Delphi process. The occurrence rate of clinical protocol triggers was prospectively studied in six potential areas (\% of patients with triggering events shown; details in ESM): cardiovascular management $(88 \%)$; sedation (67\%); weaning (22\%); source control of infection (78\%); coagulopathy (6\%); and thrombocytopenia (5\%). Three protocols with frequent triggering events and rapid rates of flow through the protocol (sedation, weaning, and cardiovascular management) were developed using a modified Delphi process and tested in this prospective clinical feasibility study. Eight centers (four from the U.S., four from Europe) participated in the first two phases and six centers (three from the U.S., three from Europe) in the third phase. One center in Europe and one in the U.S. had no cardiac surgery patients, whereas one center in the U.S. had only cardiac surgery patients.

\section{Baseline period}

Severity of illness (SAPS II score without the Glasgow Coma Scale component due to the potential for sedation influence) and crude outcomes (length of stay, intensive care unit and hospital outcome) were recorded for 4 weeks before protocol use for all patients eligible for the protocols. In addition, protocol-related outcome variables (time without mechanical ventilation and time without inotropes/vasopressors) were recorded. Thereafter, 2 weeks were used to train the research staff in the use of the protocols and software tools. In parallel, the study outline, execution, and the protocols' contents were explained to the medical and nursing staff. The research staff 
provided hands-on training in application of the protocol software. This training took place before the intervention.

\section{Intervention period}

Over a 4-week period, the protocols were applied in patients who fulfilled the inclusion criteria. Due to availability, the computerized systems were used in a maximum of four patients simultaneously in each unit on a "first-come, first-served" basis. The path through the protocols, the flow rate through the protocols (number of protocol steps per unit of time as an indicator of the rate at which interventions are applied to solve problems in care processes), time spent in and re-entries into protocols, and the occurrence rate and causes of leaving protocolized care were assessed. Severity of illness, organ failures, crude outcomes, and protocol-related outcomes (time without mechanical ventilation and time without inotropes/vasopressors) were recorded.

The protocol software was used for the first 7 days. Afterward, continued protocol use in paper format was encouraged but not verified.

\section{Control period}

After a 2-week washout period, control data were collected for 4 weeks for all patients eligible for the protocols, but no protocols were used. Severity of illness, organ failures, crude outcomes, and protocol-related outcomes (time without mechanical ventilation and time without inotropes/vasopressors) were recorded.

For all periods, the length of stay and outcome data were collected until 30 days, discharge, or death, whichever occurred first.

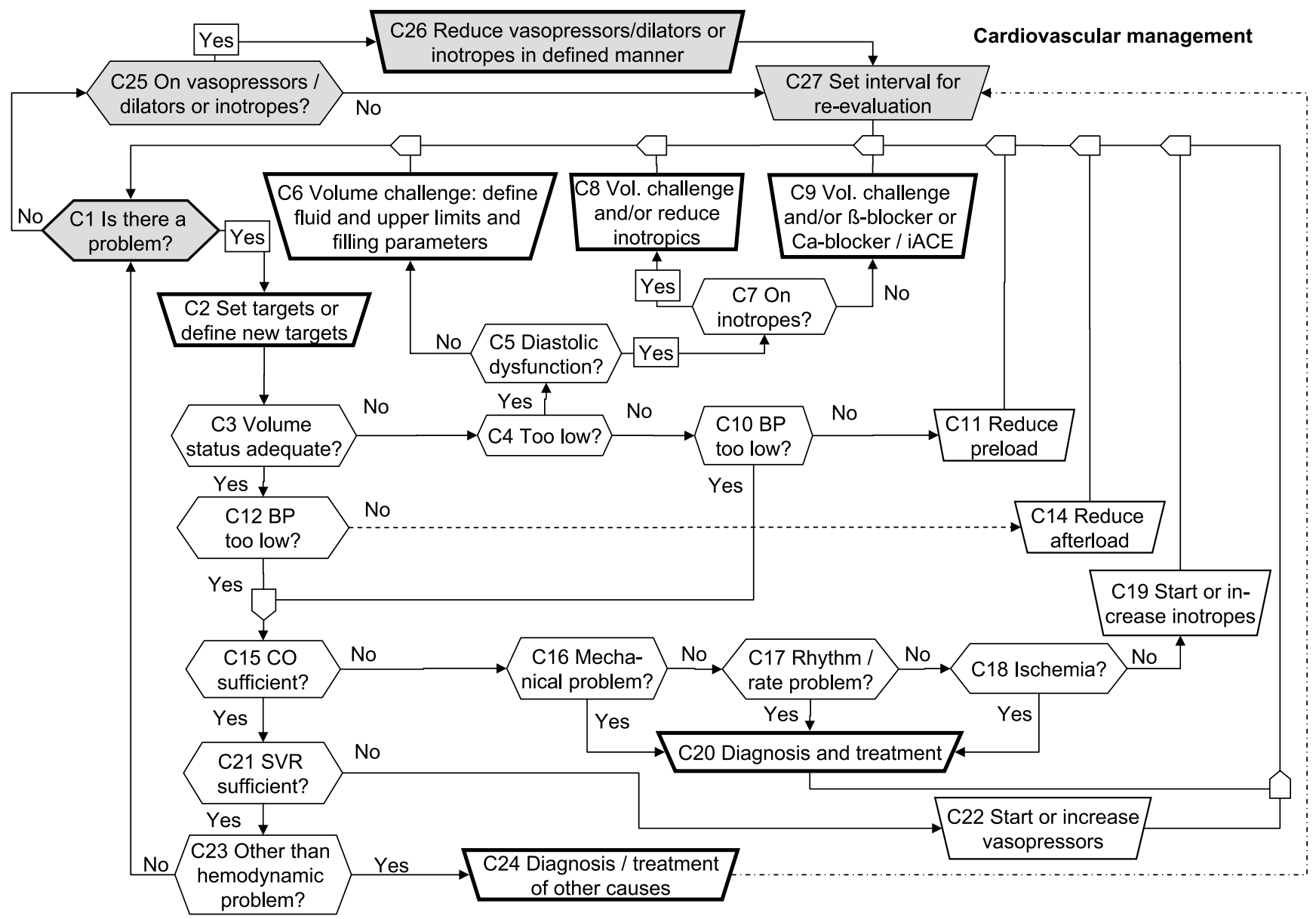

Fig. 1 Cardiovascular management protocol. The protocol consisted of a passive observation part (grey flow chart symbols: no hemodynamic problems, C1-C25-C26-C27-C1) and an active treatment part (white flow chart symbols). Hypovolemia loop: C1-C3 to C6C9; hypervolemia loop: $\mathrm{C} 1-\mathrm{C} 3$ to $\mathrm{C} 11$; (high) afterload loop: $\mathrm{C} 1$ to C12-C14; inotropes loop: $\mathrm{C} 1$ to $\mathrm{C} 15-\mathrm{C} 19$; vasopressors loop: $\mathrm{C} 1$ to
C21-C22. Physician interaction needed in defining targets and reevaluation interval $(\mathrm{C} 2, \mathrm{C} 27)$, in defining volume challenge $(\mathrm{C} 6, \mathrm{C} 8$, C9), in diagnostics (C20, C24), and in primary selection of the drug or other specific intervention $(\mathrm{C} 9, \mathrm{C} 11, \mathrm{C} 14, \mathrm{C} 19, \mathrm{C} 22) . \mathrm{BP}$, blood pressure; $C O$, cardiac output; $i A C E$, angiotensin converting enzyme inhibitor 
Software tools

A prototype computerized tool (Deio, Kuopio, Finland) containing the protocols and the information needed to define therapeutic triggers, goals of therapy, and the flow of the patient through the protocol was applied for patient care at the bedside.

\section{Protocol application}

Each protocol (Figs. 1-3) included a passive observation part and an active part consisting of several treatment loops. The clinical problems requiring interventions and the variables and clinical findings available to guide treatment were defined, but the interpretation of a clinical problem (e.g., hypovolemia, presence of pain), specifics of the intervention (e. g., selection of vasoactive or sedative drug), and therapeutic targets (e.g., blood pressure, level of sedation) were defined by the clinician in charge.

\section{Inclusion criteria}

\section{Cardiac surgical patients}

All patients undergoing either coronary artery bypass surgery, valve surgery, or a combination of both were included.

\section{Septic patients}

All patients with clinically evident sepsis on admission or within the first $24 \mathrm{~h}$ in the ICU, including clinical evidence of infection and at least two of the following signs, were included: fever or hypothermia ( $>38$ or $<36^{\circ} \mathrm{C}$ ); tachycardia ( $>90$ beats/min); tachypnea [ $>20$

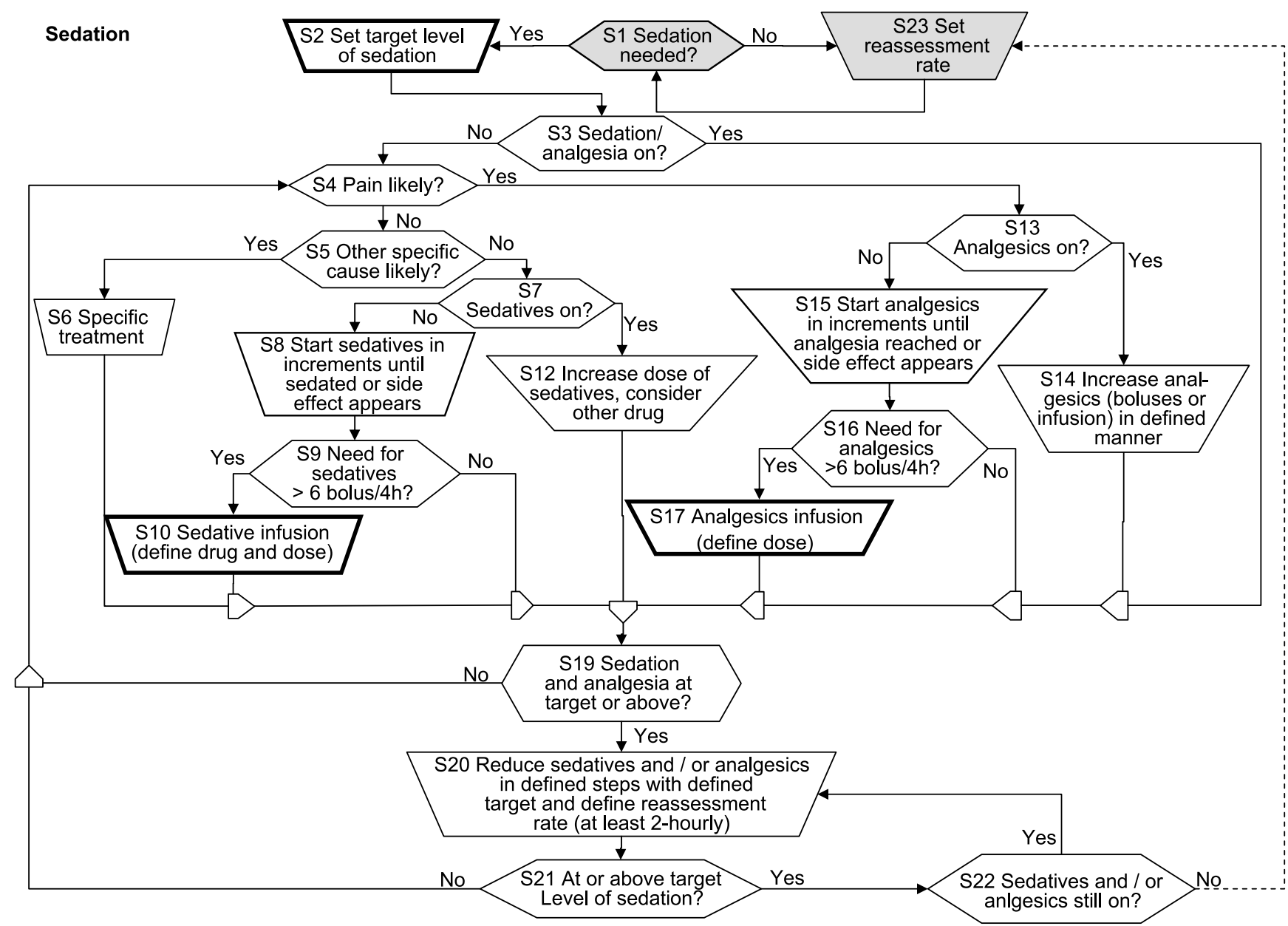

Fig. 2 Sedation protocol. The protocol consisted of a passive observation part (gray flow chart symbols: no need for sedation, S1-S23$\mathrm{S} 1$ ) and an active treatment part (white flow chart symbols). Analgesia loop: S1-S4 to S13-S19; sedation loop: S1-S4 to S5-S19; re- duction of sedatives/analgesics loop: S19-S22. Physician interaction needed in setting the target and reassessment rate (S2, S20, S23), and in selecting the drug and dose and prescribing an infusion (S8, S10, S15, S17) 
Fig. 3 Weaning protocol. The protocol consisted of a passive observation part ( gray flow chart symbols: not ready for weaning, W1-W2-W24-W25-W1) and an active treatment part (white flow chart symbols). Spontaneous breathing trial loop: W1-W6 to W7-W13b; pressure support loop: W1-W6 to W14-W21-W13b. SBT spontaneous breathing trial; $P S V$, pressure support ventilation; $A T C$, automatic tube compensation. Physician interaction needed in starting (W2, W24, W25), in defining the method (W6, W20), and in extubation (W13a)

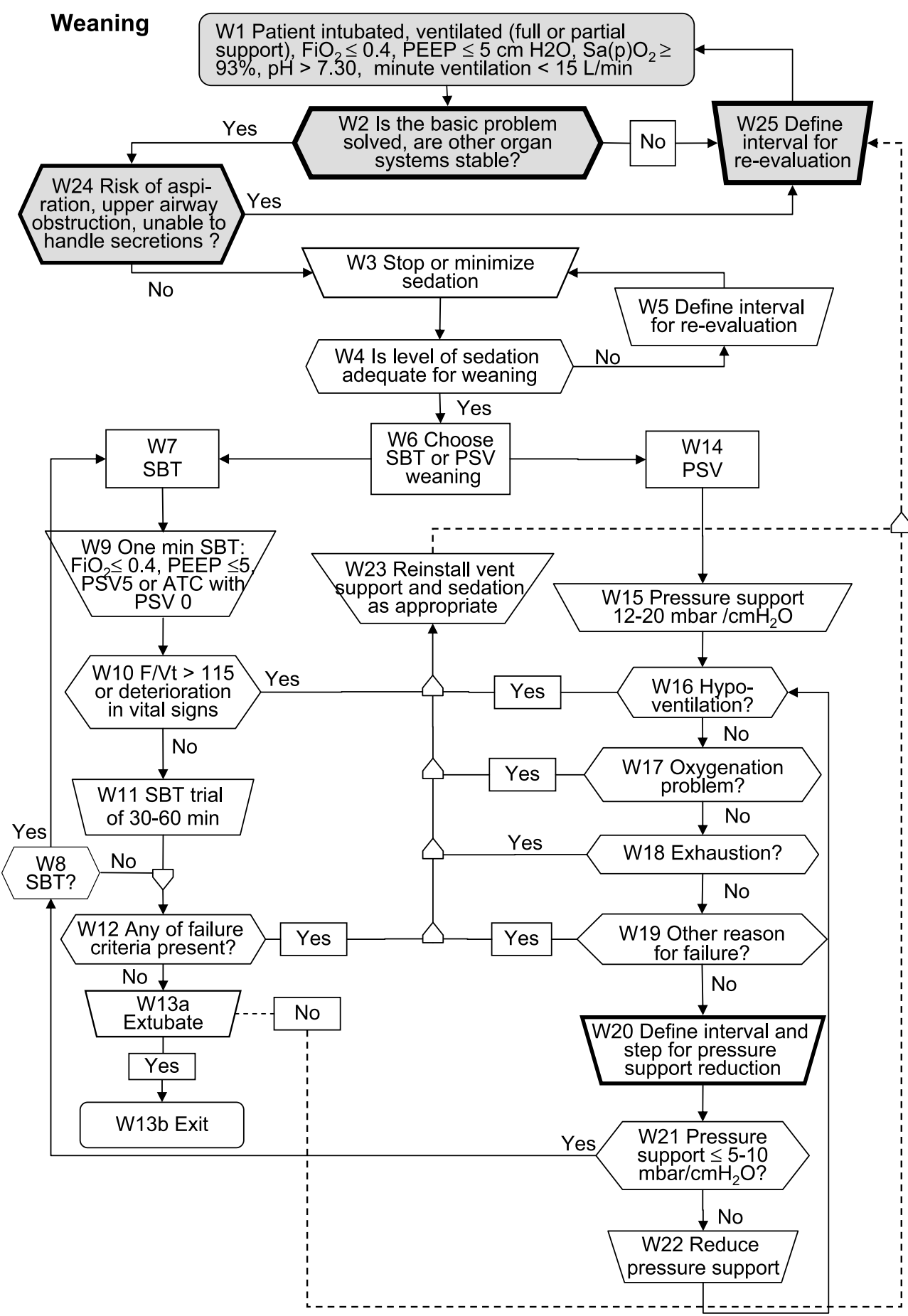

breaths $/$ min or $\mathrm{PaCO} 2<32 \mathrm{mmHg}(<4.3 \mathrm{kPa})]$; or leukocytosis/leukopenia $\left(>12,000\right.$ or $\left.<4,000 \mathrm{cu} / \mathrm{mm}^{3}\right)$.

\section{Statistical analysis}

Descriptive statistics were used to characterize the patient groups and the use of protocols. All statistics refer to the intention-to-treat population of patients treated using the computerized tool for protocols. The use of protocols as a percentage of time in the ICU was calculated for the time eligible for study protocol usage, i. e., for the actual length of stay, if less than 7 days, or as a percentage of the 7 days eligible for the use of protocols, if the stay was longer. The effect of center, geographic area (Europe vs. U.S.), and patient group (sepsis vs. cardiac surgery), on the patient 
flow rate through the protocol was tested by evaluating differences in the slope of the regression between time spent in protocol and the number of protocol steps using the general linear model. Normal distribution of the variables was tested, and logarithmic transformations were performed where necessary. The regression residual statistics and interaction terms were calculated and tested. Nonparametric tests were used for not normally distributed variables. Proportions were compared using the chi-square test. A $p$-value of less than 0.05 was considered as statistically significant. All statistical analyses were performed using SPSS for Windows software (version 12.01, SPSS, Chicago, Ill.).

\section{Results}

No significant differences were observed between the baseline, protocol, and control periods in age, severity of illness, length of ICU stay, or hospital outcome (Table 1).

The protocol software tool was used to treat 174 cardiac surgery and 41 septic patients (Fig. 4). The SAPS II scores of cardiac surgery patients were higher and the length of stays were longer in the U.S. centers than in the European centers, and there was a center-continent interaction for SAPS II $p<0.001$, for length of stay $p=0.004)$. No significant differences were observed in the septic patients. (For center-specific data see ESM, Table ES1.)

\section{Protocol usage}

Protocol software tool usage was analyzed from the beginning of the active treatment part of the protocol. Most patient time was spent in the active part of the protocol (Table 2). At least one protocol was used for 73 and $62 \%$

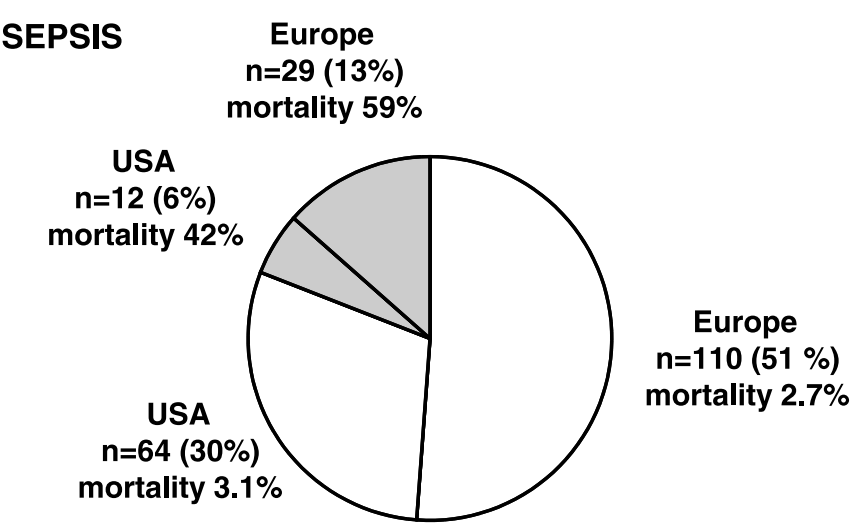

\section{CARDIAC SURGERY}

Fig. 4 Distribution of patients $(n=215)$ between the two groups (white slices: cardiac surgery; gray slices: sepsis) and geographical areas (Europe and U.S.), and their respective hospital mortalities

of the study duration in the cardiac surgery and septic patients, respectively. All three protocols were used in most $(>90 \%)$ of the cardiac surgery patients, and all three simultaneously in $85 \%$ (Table 2).

The most common combination of active protocols in the septic patients was cardiovascular management and sedation. The cardiovascular management protocol was used in $83 \%$ of septic patients, the sedation protocol in $66 \%$, and the weaning protocol in $61 \%$.

Flow rate through the protocols

The number of protocol steps per unit of time was similar in the two patient groups (Fig. 5). Significant differences between centers $(p=0.001)$ and continents $(p<0.001$; Europe vs. U.S.) were observed only for the weaning

Table 1 Patient age, intensive care unit and hospital mortality, length of intensive care unit stay, and severity of illness in baseline, protocol, and control periods

\begin{tabular}{|c|c|c|c|c|c|c|}
\hline & $\begin{array}{l}\text { Patients } \\
(n)\end{array}$ & $\begin{array}{l}\text { Age } \\
\text { (years) }^{\text {a }}\end{array}$ & $\begin{array}{l}\text { ICU mortality } \\
(\%)\end{array}$ & $\begin{array}{l}\text { Hospital } \\
\text { mortality }(\%)\end{array}$ & $\begin{array}{l}\text { ICU length } \\
\text { of stay (days) }\end{array}$ & SAPS II ${ }^{b, c}$ \\
\hline \multicolumn{7}{|l|}{ Sepsis } \\
\hline Baseline & 42 & $62(14)$ & 42.9 & 51 & $3.0(1.5-8.8)$ & $41(30-55)$ \\
\hline Intervention & 54 & $60(17)$ & 38.9 & 48 & $6.3(1.8-12.1)$ & $46(33-58)$ \\
\hline Control & 31 & 59 (19) & 25.8 & 32 & $4.4(2.1-10.1)$ & $43(31-56)$ \\
\hline Outcome baseline + control & & & 35.6 & 42 & & \\
\hline \multicolumn{7}{|l|}{ Cardiac surgery } \\
\hline Baseline & 198 & $65(11)$ & 2.0 & 3.5 & $0.9(0.8-1.9)$ & $28(23-33)$ \\
\hline Intervention & 220 & $65(11)$ & 2.2 & 2.2 & $0.9(0.8-1.6)$ & $27(22-32)$ \\
\hline Control & 208 & $65(11)$ & 1.4 & 2.9 & $0.9(0.8-1.6)$ & $26(21-31)$ \\
\hline Outcome baseline + control & & & 1.7 & 3.2 & & \\
\hline
\end{tabular}

Statistics: center-continent interaction for SAPS II $(p=0.000)$ and for length of stay $(p=0.004)$ in the cardiac surgery patients, no significant differences in septic patients; ${ }^{a}$ Mean + SD; ${ }^{b}$ Median (IQ range); ${ }^{c}$ The SAPS II scores were calculated without the Glasgow Coma Scale component 
Table 2 Number of patients in the individual protocols and their different combinations

\begin{tabular}{|c|c|c|c|c|}
\hline & $\begin{array}{l}\text { Cardiac surgery } \\
\text { No. of } \\
\text { patients }(\%)\end{array}$ & $\begin{array}{l}\text { Time in } \\
\text { active part }(\%)\end{array}$ & $\begin{array}{l}\text { Sepsis } \\
\text { No. of } \\
\text { patients }(\%)\end{array}$ & $\begin{array}{l}\text { Time in } \\
\text { active part }(\%)\end{array}$ \\
\hline $\begin{array}{l}\text { Cardiovascular management } \\
\text { Sedation } \\
\text { Weaning } \\
\text { Cardiovascular management + sedation } \\
\text { Cardiovascular management + weaning } \\
\text { Cardiovascular management + sedation + weaning } \\
\text { Sedation + weaning }\end{array}$ & $\begin{array}{l}168(97) \\
162(93) \\
164(94) \\
156(90) \\
159(91) \\
147(85) \\
148(85)\end{array}$ & $\begin{array}{l}74 \\
73 \\
83\end{array}$ & $\begin{array}{l}34(83) \\
27(66) \\
25(60) \\
27(66) \\
21(51) \\
18(44) \\
20(49)\end{array}$ & $\begin{array}{l}55 \\
82 \\
84\end{array}$ \\
\hline
\end{tabular}

protocol (for detailed statistics see ESM, Table ES2), which contained two alternative strategies (pressure support weaning or spontaneous breathing trials). This was associated with a more frequent use of the pressure support weaning loop (Fig. 3; details of loops in ESM) in Europe (in $98 \%$ of patients) vs. the spontaneous breathing trial in the U.S. (in 79\% of patients). The pressure support loop of the weaning protocol contained more steps than the spontaneous breathing trial loop.

\section{Use of protocol components and achievement of targets}

In cardiac surgery patients, the hypovolemia loop ( $\mathrm{C} 1-\mathrm{C} 3$ to $\mathrm{C} 6-\mathrm{C} 9$; Fig. 1) and tapering of vasoactive agents $(\mathrm{C} 1$ to $\mathrm{C} 25-\mathrm{C} 26$; Fig. 1) were by far the most commonly used components of the cardiovascular protocol (Fig. 5). In septic patients, the hypovolemia loop, the vasopressor loop (C1 to C21-C22, Fig. 1), and tapering of vasoactive agents were the most commonly used protocol components (Fig. 6). For the cardiovascular management protocol, the following mean target levels were observed: mean arterial blood pressure at least $63 \mathrm{mmHg}$; urinary output more than $56 \mathrm{ml} / \mathrm{h}$; lower limit for cardiac index $2.11 / \mathrm{min} \mathrm{m}^{-2}$; CVP at least $11 \mathrm{mmHg}$; CVP not more than $16 \mathrm{mmHg}$; PAOP at least $13 \mathrm{mmHg}$; PAOP not more than $18 \mathrm{mmHg}$; and $\mathrm{SvO} 2$ at least $63 \%$.

Most of the loops were entered and re-entered one to three times ( $>80 \%$ of the patients), except the tapering of vasoactive agents loop, where $42 \%$ of the patients required four or more entries and re-entries (for details see ESM, Fig. ES1). Hemodynamic stability was achieved in $26 \pm 18$ min (mean \pm SD) in the cardiac surgery patients and in $24 \pm 18 \mathrm{~min}$ in the septic patients. The hemodynamic targets were not reached in $9(5 \%)$ cardiac surgery patients and in $4(12 \%)$ septic patients.

The sedation protocol was initiated in $93 \%$ of the cardiac surgery patients and $66 \%$ of the septic patients. Sedation scores (Ramsay score or RASS score) were used to set sedation targets and to monitor the depth of sedation, whereas pain was assessed clinically. The target levels of sedation were reached in $96 \%$ of the cardiac surgery pa-

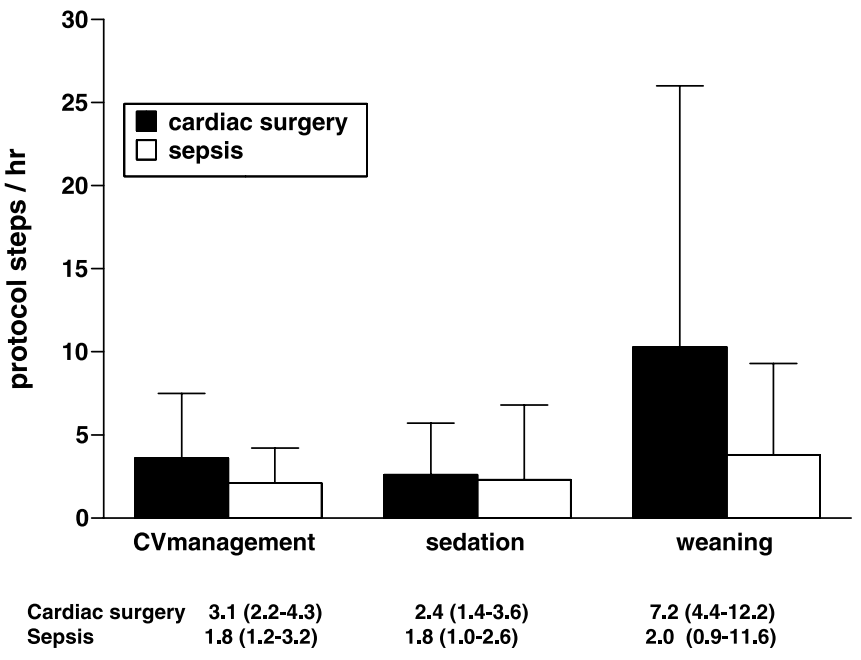

Fig. 5 Flow rate through the protocols (protocol steps/h, mean + SD; CVmanagement: cardiovascular management protocol). In addition, median (interquartile range) is indicated under the $x$-axis for each protocol. (Statistics: cardiac surgery vs. sepsis n. s. for all protocols)

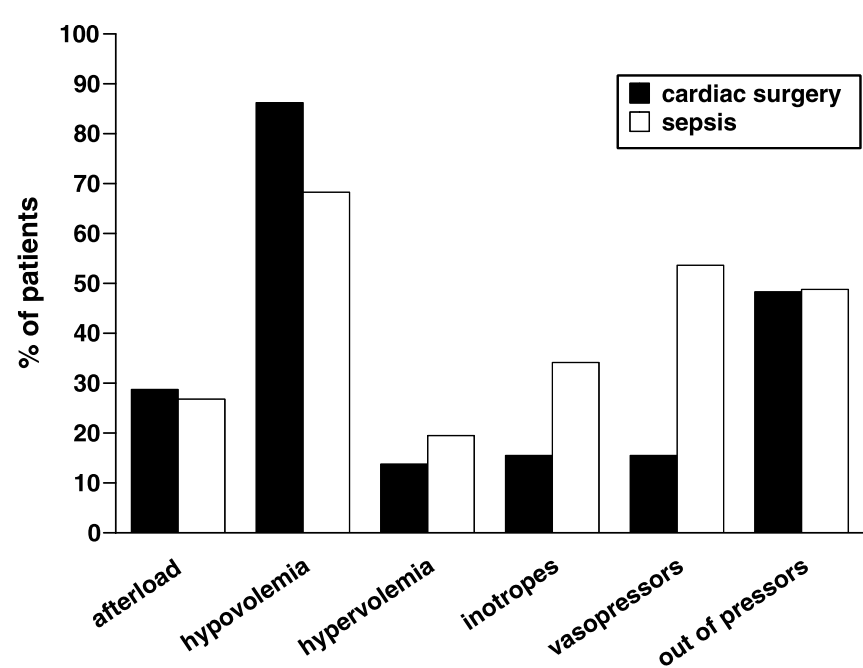

Fig. 6 Flow through the loops of cardiovascular management protocol: percentage of patients entering the loops in each patient group 
tients and $93 \%$ of the septic patients. The time needed to reach the targets was $2.4 \pm 0.2 \mathrm{~h}$ (mean $\pm \mathrm{SD}$ ) for the cardiac surgery patients and $3.6 \pm 0.2 \mathrm{~h}$ for the septic patients.

For weaning, progressive reduction of pressure support was used as the method of choice in $69 \%$ of patients; the European centers used pressure support almost exclusively (98\%), whereas the U.S. centers predominantly used the spontaneous breathing trial (78\%). Weaning was started in $94 \%$ of the cardiac surgery patients and $60 \%$ of the septic patients (Table 2), and 89 and $17 \%$, respectively, were extubated during the use of the software tool. The median time from start of weaning to extubation was $89 \mathrm{~min}$ (interquartile range 44-154 min) for the cardiac surgery patients and $96 \mathrm{~min}$ (interquartile range 56-205 min) for the septic patients. In cardiac surgery patients, the time from start of weaning to extubation was shorter in the U.S. centers (European median $101 \mathrm{~min}$, interquartile range 49-161 vs. US: $65 \mathrm{~min}, 40-120 \mathrm{~min}, p=0.036$ ), whereas time from admission to extubation was similar (European: median $542 \mathrm{~min}$, interquartile range $359-822 \mathrm{~min}$; U.S.: $570 \mathrm{~min}, 311-744 \mathrm{~min})$. Due to the small number of weaned septic patients, no continent or center effects were analyzed.

Five patients of those treated with the protocol software tool needed to be reintubated after having been extubated: one cardiac surgery patient in the U.S. and two in Europe, and two septic patients in Europe. All these patients had been weaned with the progressive reduction of pressure support approach. Of those patients for whom the protocol software tool was not available, five patients were reintubated ( 3 of 46 cardiac surgery patients and 2 of 13 septic patients, all in Europe). The overall reintubation rates before, during, and after protocol application were comparable (baseline 9 of 240 admissions, protocol 10 of 274 admissions, control 13 of 239 admissions).

\section{Protocol re-entries}

Re-entries after exit from the active part of the protocol (Fig. 7) were most common in the cardiovascular management protocol, where $52 \%$ of the cardiac surgery patients and $71 \%$ of the septic patients re-entered the active part of the protocol, compared with 12 and $48 \%$, respectively, in the sedation protocol and 23 and $40 \%$ in the weaning protocol.

\section{Protocol violations/premature discontinuations}

The use of protocols was discontinued in 25 patients (12\%) because of early death (4 deaths, 3 died during active protocolized treatment) or therapy limitation/withdrawal (4 patients), or need for an unrelated diagnostic or therapeutic intervention (e. g., reoperation; 17 patients). In the remaining 190 patients, protocol-driven care was applied

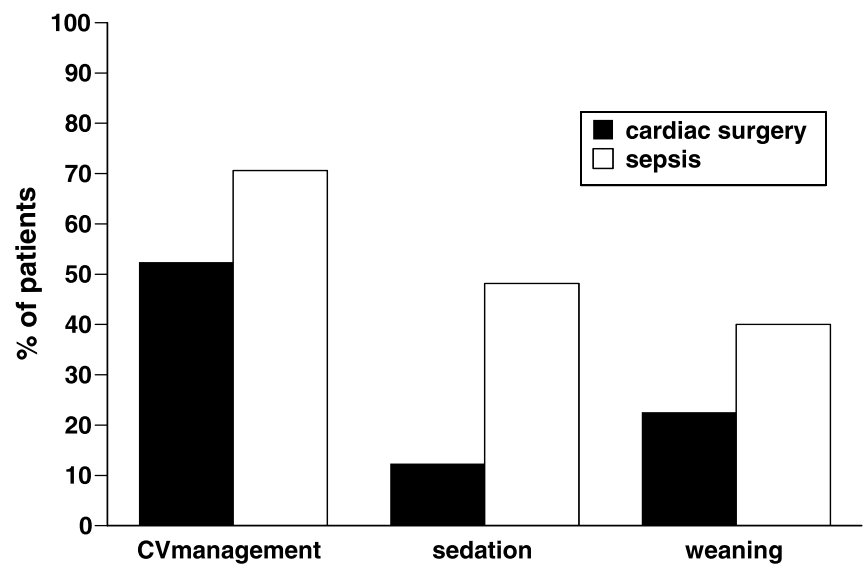

Fig. 7 Re-entries into the active part of the protocol (\% of patients). CVmanagement: cardiovascular management protocol

without violations in 155 patients (82\%); protocol use was discontinued in 12 patients $(6 \%)$ for technical or administrative reasons (software malfunction in 8 patients, patient transfer, or not specified in 4 patients); and protocol use was discontinued in 23 patients $(12 \%)$ due to the treating clinician's decision to deviate from the protocol-driven care (in 14 cardiac surgery and 9 septic patients). In 11 cases (47\% of all protocol deviations due to clinical decisions), the deviation was from the sedation protocol and was due to either an anticipated short need for sedation or start of infusion without first trying bolus sedation.

\section{Discussion}

This international, multicenter study tested in a clinical trial the feasibility and interactions among software-driven protocols for cardiovascular management, sedation, and weaning. This study demonstrated that a uniform therapeutic strategy could be formulated based on a defined sequence of logical steps, translated to treatment protocols, and then applied using software tools in the clinical management of critically ill patients in an international, multicenter setting. The use of specific interventions and care process components, the flow rate through the protocols, and perhaps most importantly, the time needed to achieve clinically relevant targets, provides core information for process analysis and performance indicators.

Although no effects on either crude or protocol-related outcomes were observed, the study was designed to assess feasibility and was not powered for outcome with these specific protocols. Accordingly, any conclusions regarding the outcome effects of the treatment strategies used in this study or regarding protocols in general should be drawn with caution. Also, the specific protocols used in 
this study should go through further validation before being integrated into routine clinical practice. Nevertheless, software-driven process control using multiple protocols was feasible, and the rarity of deviations from the protocoldriven care due to clinician disagreement suggests high acceptance.

The patient groups were selected to cover a broad range of clinical problems, a large number of patients, and two care processes with very different characteristics: rapid turnover and low mortality (cardiac surgery) and long stay and high mortality (sepsis). The selection of protocol areas was based on their relevance for both patient groups, the prospectively verified occurrence rate of triggering events, and their close interaction in clinical practice.

The high compliance rate with multiple protocols was comparable to rates reported when using only one protocol $[5,6]$. The high acceptance is probably related to several factors [14-16]:

1. Much effort was invested in reaching a consensus between the participating centers with regard to treatment strategies.

2. Specific disorders were defined in broad enough terms to allow clinical interpretation, and local preferences in monitoring, choice of drugs, and method of weaning were allowed.

3. The protocols were mostly nurse-driven but required physician interaction in specified steps.

4. The protocols focused on defining physiologic derangements rather than cut-off limits. We suggest that using this qualitative-physiologic approach instead of strict numeric therapeutic targets is advantageous, since the numeric targets for individual patients can be highly variable and depend on the underlying clinical condition.

5. An intensive training period prior to the protocol application and support in protocol use for the duration of the study were provided by the research personnel.

6. All of the participating units were either closed units run by intensive care specialists or had intensive care specialist-driven co-management.

Each protocol contained steps for which the time interval between interventions can vary. This has a poten- tially major impact on the rate of the care process. We found no differences between patient groups, centers, or continents in the number of protocol steps per time, except in weaning. The two alternative weaning strategies contained different numbers of protocol steps, which invalidates the number of protocol steps per time as a process indicator in weaning. Indeed, the preferential use of spontaneous breathing trials in the U.S. resulted in shorter times from start of weaning to extubation, fewer protocol steps per time, but similar times from admission to extubation as in the European centers.

Initial cardiovascular stabilization could be achieved rapidly in both patient groups. In contrast, several hours were needed to reach target levels of sedation. Difficulties in reaching target sedation levels despite protocols have been reported by others $[1,2]$. Once the criteria for starting weaning were reached, patients were weaned and extubated very rapidly in both groups and substantially faster in comparison with weaning times reported previously [3]. This may reflect a synergy between the three protocols.

The simultaneous use of protocols for cardiovascular management, sedation, and weaning likely reduced the between-center variation in the patient care process. The strategies of sedation, weaning, and cardiovascular management may have relevant impact on common outcome variables, such as length of ICU stay or duration of mechanical ventilation. Reducing the inherent heterogeneity between centers by simultaneous use of multiple protocols may therefore facilitate the evaluation of specific interventions targeted to influence these patient outcomes, not only at a single center as part of a performance improvement process but at many centers participating in a clinical research trial.

Care protocols can improve patient outcome only if the interventions they include are beneficial. Our results demonstrate that use of electronic protocols can facilitate care process analysis. Process flow rates, success rates, and time needed to reach clinically relevant targets can serve as performance indicators. In addition, broad aspects of patient management can be standardized in an international multicenter clinical research setting. No conclusions regarding the impact of improved process control on patient outcomes can be made on the basis of this feasibility study.

\section{References}

1. Smith CA, Corripio AB (2005) Principles and practices of automatic process control, 3rd edn. Wiley, New York

2. Kress JP, Pohlman AS, O'Connor MF, Hall JB (2000) Daily interruption of sedative infusions in critically ill patients undergoing mechanical ventilation: N Engl J Med 342:1471-1477
3. Brook AD, Ahrens TS, Schaiff R, Prentice D, Sherman G, Shannon W, Kollef MH (1999) Effect of a nursingimplemented sedation protocol on the duration of mechanical ventilation. Crit Care Med 27:2609-2615
4. Ely EW, Baker AM, Dunagan DP, Burke HL, Smith AC, Kelly PT, Johnson MM, Browder RW, Bowton DL, Haponik EF (1996) Effect on the duration of mechanical ventilation of identifying patients capable of breathing spontaneously. N Engl J Med 335:1864-1869 
5. Kollef MH, Shapiro SD, Silver P, St. John RE, Prentice D, Sauer S, Ahrens TS, Shannon W, BakerClinkscale D (1997) A randomized, controlled trial of protocol-directed versus physician-directed weaning from mechanical ventilation. Crit Care Med 25:567-574

6. The Acute Respiratory Distress Syndrome Network (2000) Ventilation with lower tidal volumes as compared with traditional tidal volumes for acute lung injury and the acute respiratory distress syndrome. N Engl J Med 342:1301-1308

7. McKinley BA, Moore FA, Sailors RM, Cocanour CS, Marquez A, Wright RK, Tonnesen AS, Wallace CJ, Morris AH, East TD (2001) Computerized decision support for mechanical ventilation of trauma induced ARDS: results of a randomized clinical trial. J Trauma 50:415-424

8. Rivers E, Nguyen B, Havstad S, Ressler J, Muzzin A, Knoblich B, Peterson E, Tomlanovich M, Early Goal-Directed Therapy Collaborative Group (2001) Early goal-directed therapy in the treatment of severe sepsis and septic shock. N Engl J Med 345:1368-1377
9. Wilson J, Woods I, Fawcett J, Whall R, Dibb W, Morris C, McManus E (1999) Reducing the risk of major elective surgery: randomised controlled trial of preoperative optimisation of oxygen delivery. Br Med J 318:1099-1103

10. Pearse R, Dawson D, Fawcett J, Rhodes A, Grounds RM, Bennett ED (2005) Early goal-directed therapy after major surgery reduces complications and duration of hospital stay. A randomised, controlled trial. Crit Care 9:R687-R693

11. Polonen P, Ruokonen E, Hippelainen M, Poyhonen M, Takala J (2000) A prospective, randomized study of goal-oriented hemodynamic therapy in cardiac surgical patients. Anesth Analg 90:1052-1059

12. McKendry M, McGloin H, Saberi D, Caudwell L, Brady AR, Singer M (2004) Randomised controlled trial assessing the impact of a nurse delivered, flow monitored protocol for optimisation of circulatory status after cardiac surgery. Br Med J 329:258

13. Ely EW, Bennett PA, Bowton DL, Murphy SM, Florance AM, Haponik EF (1999) Large scale implementation of a respiratory therapist-driven protocol for ventilator weaning. Am J Respir Crit Care Med 159:439-446
14. Scheinhorn DJ, Chao DC, StearnHassenpflug M, Wallace WA (2001) Outcomes in post-ICU mechanical ventilation: a therapist-implemented weaning protocol. Chest 119:236-242

15. Burns SM, Earven S, Fisher C, Lewis R, Merrell P, Schubart JR, Truwit JD, Bleck TP, University of Virginia Long Term Mechanical Ventilation Team (2003) Implementation of an institutional program to improve clinical and financial outcomes of mechanically ventilated patients: one-year outcomes and lessons learned. Crit Care Med 31:2752-2763

16. Trzeciak S, Dellinger RP, Abate NL, Cowan RM, Stauss M, Kilgannon JH, Zanotti S, Parrillo JE (2006) Translating research to clinical practice: a 1-year experience with implementing early goal-directed therapy for septic shock in the emergency department. Chest 129:225-232 